\title{
CFD STUDY OF USING DIFFERENT HEAT SINKS FOR ELECTRONIC EQUIPMENTS COOLING
}

\author{
A.E.Kabeel, A.Khalil, G.I.Sultan* and M.I.El-Hadary \\ Tanta University, Mechanical Engineering Department, Egypt \\ *Mansoura University, Mechanical Engineering Department, Egypt \\ E-Mail: mohamed.elhadary@f-eng.tanta.edu.eg
}

\begin{abstract}
Cooling of Electronic equipment's is an attractive research area in engineering applications. Continued minimization of electronic system has resulted in dramatic increase in the amount of heat generated per unit volume, The aim of this study is to use computational Fluid Dynamics in order to draw a CFD model for forced cooling conjugate heat transfer analyses in heat generating electronic systems and compare between a collection of actual commercial heat sinks different from in geometry, material, and number of fins .A complete computer chassis model with heat sinks and fans inside was created and parametric analyses were performed to compare the effects of different turbulence models, mesh resolutions, and radiative heat transfer. The CFD software was used, ANSYS Icepack $\mathbf{1 8 . 0}$ for preprocessing and fluent for solution and post processing. The road map was applied to five different heat sinks and another three heat sink as a validation modeled into the full chassis. Numerical results were compared with the available experimental data and they were in good agreement.
\end{abstract}

\section{INTRODUCTION}

Electronics cooling encompasses thermal design, analysis and experimental characterization of electronic systems (Heat Sinks) as a discrete discipline with the product creation process for an electronics product, or an electronics sub-system within a product.

Heat sinks are devices that are used to increase surface area of heat transfer of electronic components available for air cooling, helping to lower the components case temperature. Fans are used to increase the cooling air flow rate.

Thermal design and analysis is performed using hand calculations or spreadsheets, based on design rules or heat transfer correlations. Computer-aided engineering tools such as computational fluid dynamics are also used. Software for electronics cooling includes Ansys' Icepack.

Computational fluid dynamics (CFD) codes are widely used as a tool of thermal analysis. CFD solutions of high spatial and temporal resolutions can be obtained on a desktop computer or even a laptop. However, CFD-based thermal analysis is not necessarily easy to perform where the object of analysis is geometrically complex

\section{LITERATURE}

$\mathrm{Yu}$ and Webb [1] used Icepak to analyze the flow and heat transfer inside a desktop computer which had an $80 \mathrm{~W}$ CPU. The design was for a total chassis power of $313 \mathrm{~W}$. In their model, motherboard, PCI/AGP cards and memory were modeled as zero thickness rectangular plates with heat generated uniformly on the component side. The HDD and DVD were modeled as solid blocks generating a specified amount of heat inside the volume. Finally, the power supply and the CPU-heat sink were modeled as a volume resistance. In this study, the key design parameter was to minimize chassis air flow requirements.

Eveloy, Rodgers and Hashmi [2] used Flotherm software to provide a perspective on the current capabilities of CFD as a design tool to predict component temperature on printed circuit boards. Their computations predicted the component operating temperature in an accuracy range of $3{ }^{\circ} \mathrm{C}$ to $22{ }^{\circ} \mathrm{C}$, with up to $35 \%$ error. They suggested that component junction temperature would need to be measured experimentally when used for strategic product design decisions. They thought that the visualization in the early design phase to identify aerodynamically sensitive regions on the board, where temperature distributions should be handled with care. The present study makes use of CFD for the conjugate heat transfer simulations in a whole computer chassis with an aim of drawing a road map based on experiences gained during the process. ANSYS Icepack is used for preprocessing

Ozturk [3], Computational Fluid Dynamics, which has taken its position in the thermal design of electronic packages, was used in order to draw a CFD road map for forced cooling conjugate heat transfer analyses in heat generating electronic systems. The main sources of error in CFD analyses arise from inappropriate numerical models including turbulence models, radiation modeling and discretization schemes, insufficient grid resolution, and lack of convergence. A complete computer chassis model with heat sinks and fans inside was created and parametric analyses were performed to compare the effects of different turbulence models, discretization schemes, mesh resolutions, convergence criteria, and radiative heat transfer. Two commercially available CFD software packages were used, ANSYS Icepack for preprocessing and FLUENT for solution and post processing. The road map was applied to three different heat sinks modeled into the full chassis. Numerical results were compared with the available experimental data and they were in good agreement. 
Khan, J. R., and Yovanovich [4] analyze the performance of a cylindrical pin-fin heat sink in laminar forced convection. The mathematical models are presented for predicting thermal and hydraulic resistances for both in-line and staggered arrangements. Analytical/empirical correlations of friction and heat transfer coefficients are used in the analysis. The analyses are performed by using parametric variation of resistances. The effects of thermal joint, spreading, and contact resistances as well as the thermal conductivity on the overall thermal performance are examined. For a given size and heat load, it is observed that the overall performance of a pin-fin heat sink depends on a number of parameters including the dimensions of the pinfins, pin density, longitudinal and transverse spacing's, interface material, location and size of heat sources, method of manufacturing, type of heat sink material, approach velocity, and arrangement of pins. It is also observed that the thermal resistance decreases whereas pressure drop increases with an increase in approach velocity, pin diameter, and pin density.

Ece Ayli, Caner Turk, and Selin Aradag [5] designed vortex promoters for cooling of electronic equipment. Different shapes of vortex promoters are used in the experimental study for turbulent flow and the results are used to validate the results of a previous computational work performed by the authors. Another aim is to choose an appropriate promoter and promoter location which provides best turbulence effects and most effective cooling. Temperature values are measured with thermocouples at several Monitoring locations. The results show that the most effective vortex promoter for cooling is triangular type of promoter for the flow conditions tested.

Snehal Saste, Doshi, Khalate, Shailesh Jedhe, Nupura Yadav [6] had Proposed thermal analysis of a server system, and made effort to lower the maximum temperature in the CPUs by changing CPU heat sink design. The server computer of form factor SSI EEB is modelled in detail and is analyzed by using commercial computational fluid dynamics (CFD) software packages Icepack and Fluent. To optimize parameters of heat, sink parameters the CFD simulations are performed for maximum dissipation of heat. Little iteration are performed by changing geometry and material of heat sinks number of fins of heat sinks and changing the fan positions on computer chassis. The optimum grouping of parameters and results will be compared with the commercially available heat sink.

\section{CFD Simulation APPROACH}

The simulation approach for the full computer chassis model is discussed briefly in this section.

\section{III.1 Computer Chassis Model}

The model studied consists of the following objects: The details related to the model and the software can be found in the work of Elhadary (2019) and the icepak 18.0 user guide respectively.

Computer chassis
It defines the computational domain. No mesh is generated outside the computer chassis.

\section{CPU}

It is the main heat source in the model, The CPU is modeled as which dissipates $95 \mathrm{~W}$ for each model. The CPU dimensions are equivalent to a commercially available CPU. CPU Fan

The fan is modeled as $2 \mathrm{D}$ circular, fixed flow rate equals 83 cfm.

\section{Heat sink}

There are two simple aluminum heat sinks. They are much smaller than the one on the CPU.

\section{Mainboard}

It is the main card on which the CPU, chipset and other cards are placed. It is modeled with its thickness.

\section{Grilles}

The computer cases have small holes on them, which make air inlet or outlet possible. Since modeling these holes make the model computationally expensive, lumped parameter models are used again. They act like resistance to the flow according to the specified free area ratios.

\section{DVD and hard drives}

Hard disks, DVD-Rom are modeled as 3D blocks with heat dissipation. While they may be neglected with respect to their heat dissipation rates, they are included since they affect air flow in the system.

\section{Ram Cards \& PCIs}

There are two ram cards and two PCIs are placed on the main board. They are also heat sources.

\section{Power supply}

The modeling of the power supply is totally different than other components in this study. The power supply is a resistance to flow; therefore, it is modeled using resistance feature in Icepack. The effect of resistance is modeled as a pressure drop through its volume. However; there are two possible methods to calculate the pressure drop, approach velocity and device velocity methods which only differ by a factor which is called the free area ratio. As a general practice in icepack, for laminar flows a linear velocity relationship should be selected $(n=1)$, whereas a quadratic relationship $(n=2)$ should be selected for a turbulent flow. Fig. 1 shows the components of the chassis. The chassis is modeled using dimensions of a common ATX chassis $(\mathrm{L} \times \mathrm{W} \times \mathrm{H}=380 \mathrm{~mm} \times 335 \mathrm{~mm} \times 90 \mathrm{~mm})$, and all the components inside the chassis are standard-sized components that are used in most desktop computers. During modeling, exact dimensions of the components were either sourced from manufacturer specifications or obtained by measurement. Fig. 2 and Fig 3 show the detailed model of one of the heat sinks considered. Geometric details and measurements of the heat sinks were obtained from manufacturers. 


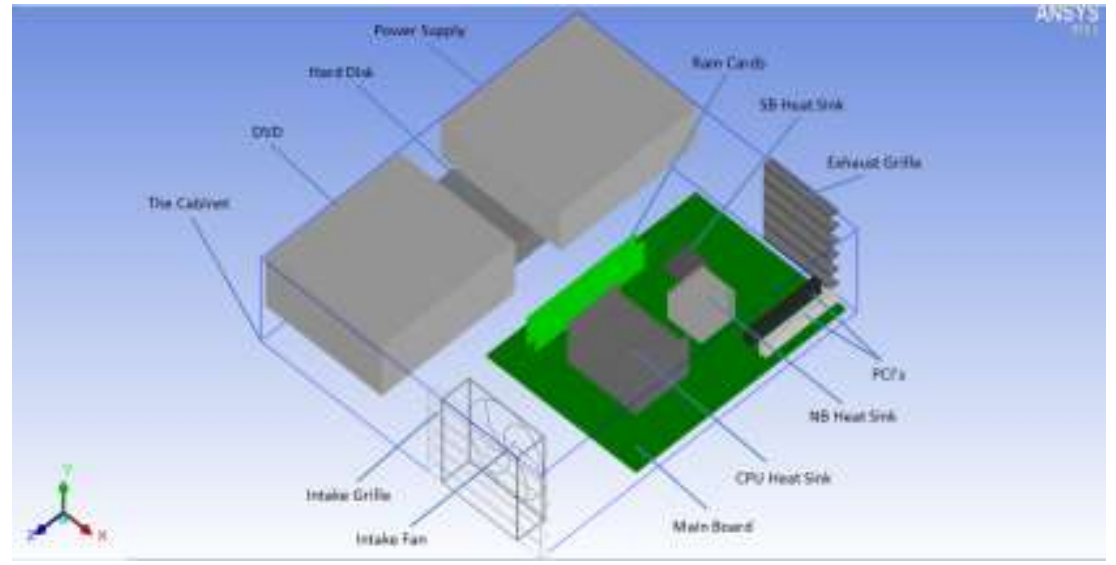

Figure 1. Computer chassis model $(\mathrm{L} \times \mathrm{W} \times \mathrm{H})$ in $(\mathrm{Z} \times \mathrm{X} \times \mathrm{Y})$ directions.

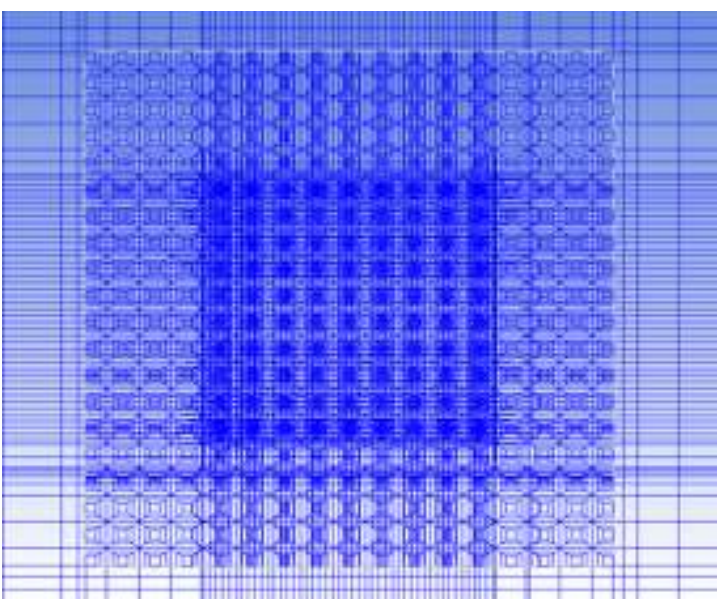

Figure 2. Surface grid on one of the CPU heat sinks.

\section{III.2 Mesh Generation}

The mesh is the key component of a high quality solution. In our simulations hexahedral unstructured meshing is used. It is important to have a good mesh to have an accurate solution. There are some general guidelines called rules of QRST, standing for, Quality, Resolution, Smoothness and Total cell count. Mesh resolution is determined by showing grid independence with respect to coarser and finer meshing. Mesh quality is automatically determined by ANSYS Icepack considering face alignment, skewness, aspect ratio and cell size. Smoothness in mesh distribution is assured by keeping the growth rate of the cells from finer mesh to coarser mesh below 20\%. Considering our computer resources, the total number of cells generated is kept around one million for the entire model.

\section{III.3 Governing Equation}

The three-dimensional steady-state, turbulent compressible flow in the cabinet is governed by continuity, momentum and energy equations together. The governing equations of the flow are modified according to the conditions of the simulated case. Since the problem is assumed to be steady

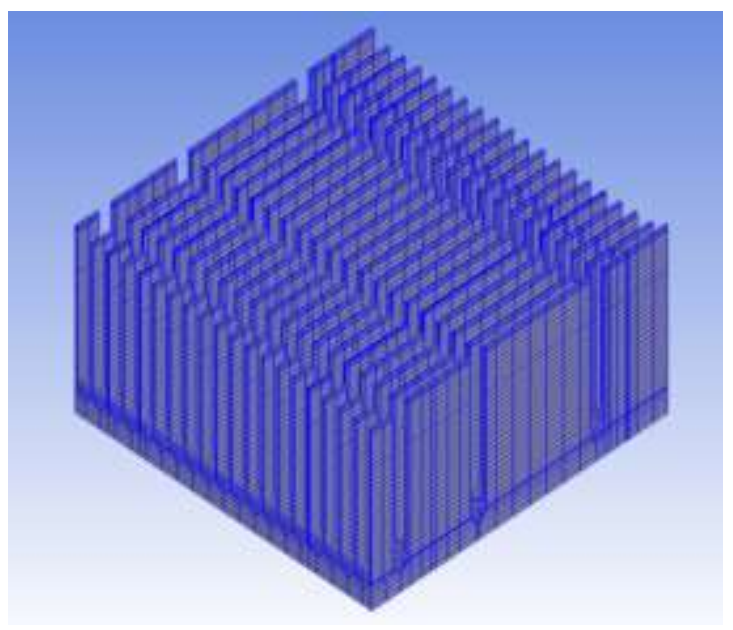

Figure 3. Non-conformal grid interface.

state with low velocities. Therefore, time dependent parameters are dropped together with the viscous dissipation term are dropped from the equations. The resulting equations are:

$$
\frac{\partial \rho}{\partial t}+\nabla \cdot(\rho \vec{V})=0
$$

$\mathrm{X}$-momentum:

$\frac{\partial(\rho u)}{\partial t}+\nabla \cdot(\rho u \vec{V})=-\frac{\partial p}{\partial x}+\frac{\partial \tau_{x x}}{\partial x}+\frac{\partial \tau_{y x}}{\partial y}+\frac{\partial \tau_{z x}}{\partial z}+\rho f_{x}$

Y-momentum:

$\frac{\partial(\rho v)}{\partial t}+\nabla \cdot(\rho v \vec{V})=-\frac{\partial P}{\partial y}+\frac{\partial \tau_{x y}}{\partial x}+\frac{\partial \tau_{y y}}{\partial y}+\frac{\partial \tau_{z y}}{\partial z}+\rho f_{y}$

Z-momentum:

$\frac{\partial(\rho w)}{\partial t}+\nabla \cdot(\rho w \vec{V})=-\frac{\partial p}{\partial z}+\frac{\partial \tau_{x z}}{\partial x}+\frac{\partial \tau_{y z}}{\partial y}+\frac{\partial \tau_{z z}}{\partial z}+\rho f_{z}$

Energy: 


$$
\frac{\partial(\rho e)}{\partial t}+\nabla \cdot(\rho e \vec{V})=-p \nabla \cdot \vec{V}+\nabla \cdot(k \nabla T)+\Phi+q
$$

$$
p=\rho R T
$$

Equation of state:

For the above equations where $\rho$ is the density; $u, v$ and $w$ are the velocity components in the $\mathrm{x}, \mathrm{y}$ and $\mathrm{z}$ respectively, $\mathrm{V}$ is the velocity vector, $f \mathrm{x}, f \mathrm{y}$ and $f \mathrm{z}$ are the body forces, $\mathrm{p}$ is pressure, $\mathrm{q}$ is the heat flux as a source term, $\mathrm{R}$ is the gas constant and $\tau$ is the shear stress which can be defined for Newtonian fluids.

\section{III.4 Boundary Conditions}

Navier-Stokes equations, turbulence model are solved for flow velocity, pressure, and temperature variables. The analysis results by taking ambient temperature $\left(\mathrm{T}_{\circ}\right)$ as $25^{\circ} \mathrm{C}$. gauge pressure $0 \mathrm{~N} / \mathrm{m}^{2}$, and radiation temperature of $25^{\circ} \mathrm{C}$.the default fluid is air. The default turbulence model of all calculations is the Algebraic turbulence model. No-slip boundary condition is applied to all walls in the domain. Therefore, at all surfaces $\mathrm{u}=\mathrm{v}=\mathrm{w}=0$. It is assumed that the system fan does not drive a flow cell around the computer chassis and the heat transfer mechanism at the chassis outer walls is natural convection.

\section{III.5 Turbulent Modeling}

The default turbulence model of all calculations is Algebraic Turbulence Model. It is a two-equation model and computationally least expensive since no extra equations are solved in addition to continuity, momentum and energy equations. However, in order to rely on the results that algebraic model gives; it should be validated with higherorder turbulence models. $\mathrm{k}-\varepsilon$ model was used as a test case. The temperature distributions and velocity fields are compared. The results show acceptable agreement Therefore, the Algebraic Turbulence Model is suitable for use.

\section{III.6 Radiation Effect}

Alpha heat sink was analyzed to investigate the radiation effects. Radiation heat transfer helped the Alpha heat sink cool by less than additional $0.5^{\circ} \mathrm{C}$. Therefore, it is concluded that radiation could be ignored for forced cooling of CPUs.

\section{Result AND Discussion}

The simulations are done using the chassis model with five different commercial heat sinks and there are three heat sinks as a validation.

The first group of results is for temperature distribution of the three heat sinks but in different chassis from the validation paper one [3].

Table 1

Maximum and minimum temperatures for the cases of the validation

\begin{tabular}{|c|c|c|c|}
\hline & Alpha & Coolermaster & Evercool \\
\hline $\mathrm{T}_{\max }(\mathrm{k})$ & $\begin{array}{c}328 \\
\left(55^{\circ} \mathrm{c}\right)\end{array}$ & $\begin{array}{c}338 \\
\left(65^{\circ} \mathrm{c}\right)\end{array}$ & $\begin{array}{c}336 \\
\left(63^{\circ} \mathrm{c}\right)\end{array}$ \\
\hline $\mathrm{T} \min (\mathrm{k})$ & $\begin{array}{c}316 \\
\left(43^{\circ} \mathrm{c}\right)\end{array}$ & $\begin{array}{c}324 \\
\left(51^{\circ} \mathrm{c}\right)\end{array}$ & $\begin{array}{c}323 \\
\left(50^{\circ} \mathrm{c}\right)\end{array}$ \\
\hline
\end{tabular}

\begin{tabular}{|l|l|l|l|}
\hline$\Delta \mathrm{T}$ & 12 & 14 & 13 \\
\hline
\end{tabular}

Table 2

Maximum and minimum temperatures for the three heat sinks

\begin{tabular}{|c|c|c|c|}
\hline & Alpha & Coolermaster & Evercool \\
\hline $\mathrm{T}_{\max }\left({ }^{\circ} \mathrm{C}\right)$ & 53.8 & 57.2 & 56.3 \\
\hline $\mathrm{T}_{\min }\left({ }^{\circ} \mathrm{C}\right)$ & 37.3 & 32.2 & 35.2 \\
\hline$\Delta \mathrm{T}\left({ }^{\circ} \mathrm{C}\right)$ & 16.5 & 25 & 21.1 \\
\hline
\end{tabular}

Group 1: Temperature distribution on heat sinks

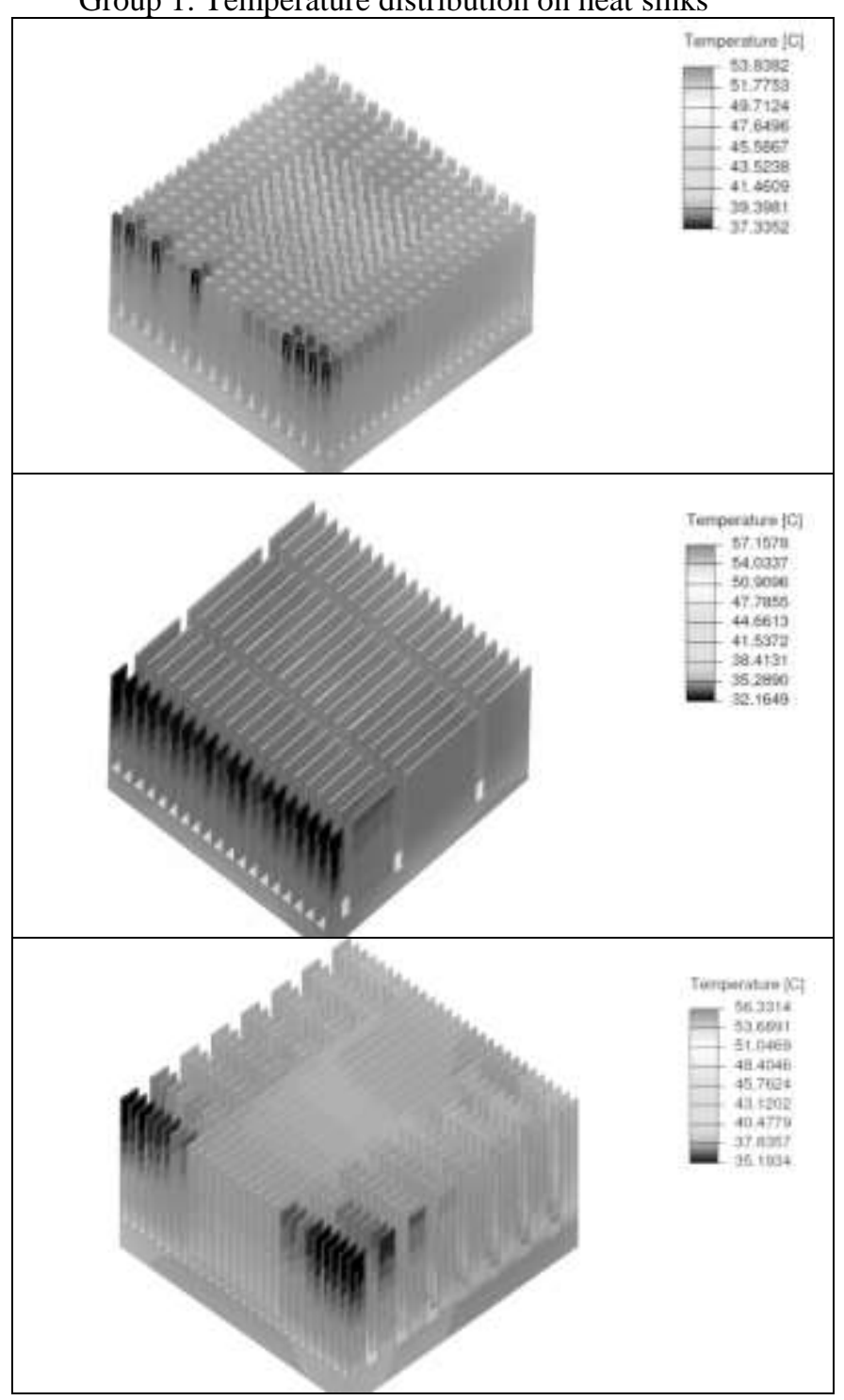

Figure 4. Temperature Distribution $\left({ }^{\circ} \mathrm{C}\right)$ on Different CPU Heat Sinks.

Alpha heat sink outperforms the other two heat sinks although the difference in conditions in the new cabinet. The second group: 
First, we assume the cabinet without cpu heat sink, its observed that some component are heating beyond their maximum limits.

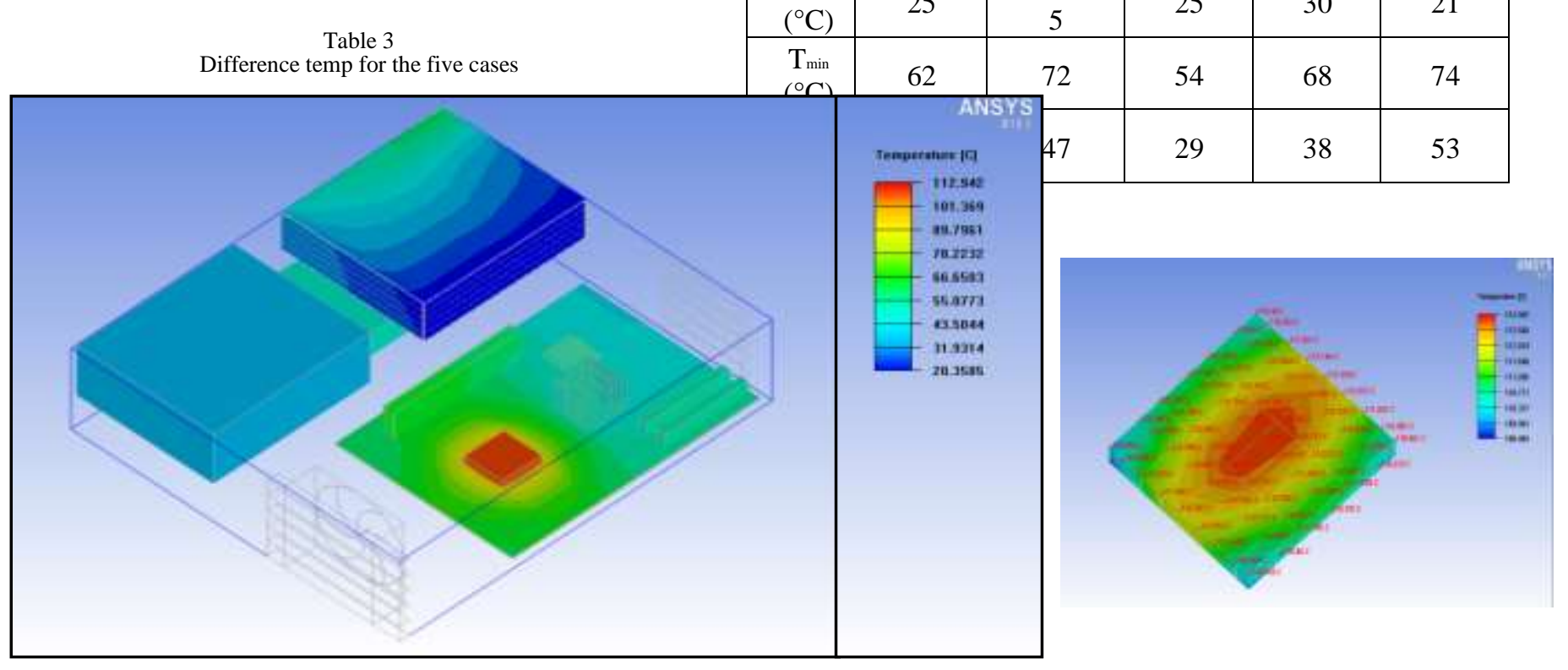

Figure 5. Cabinet without Heat Sink.

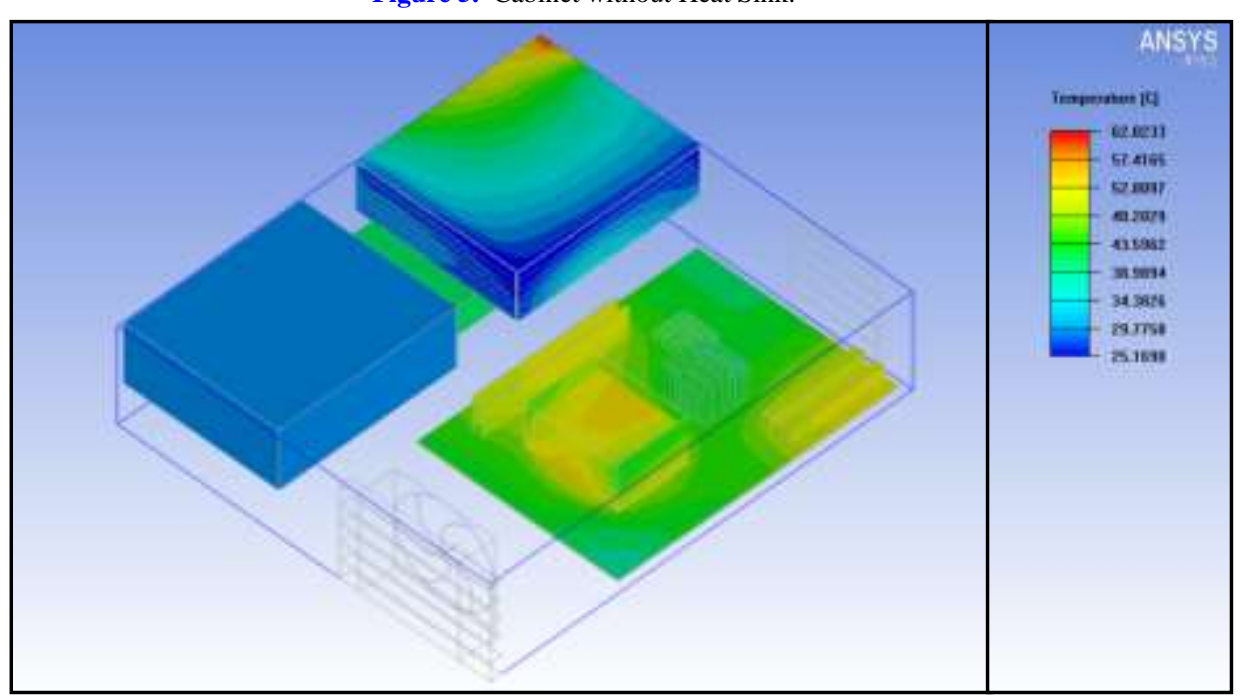

Figure 6a. Case (1).
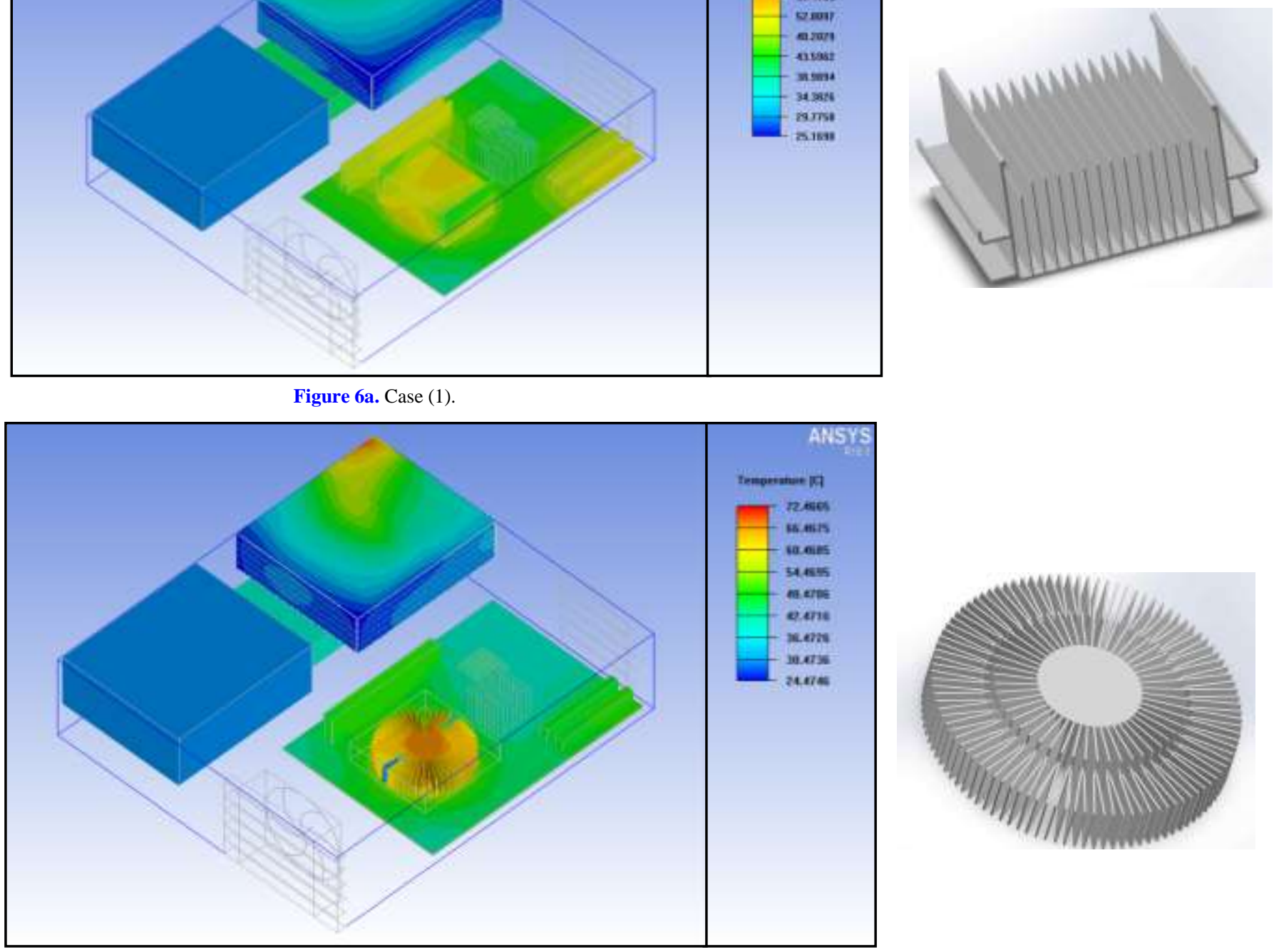
Figure 6b. Case (2).
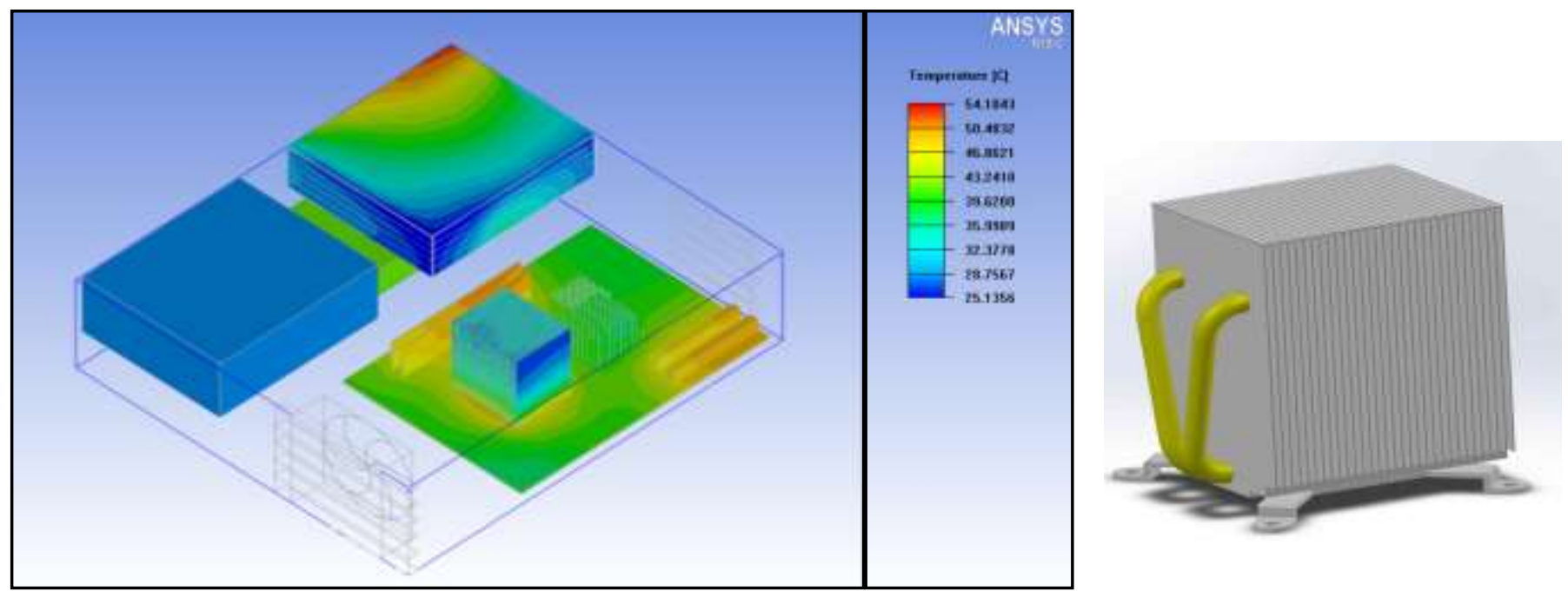

Figure 6c. Case (3).
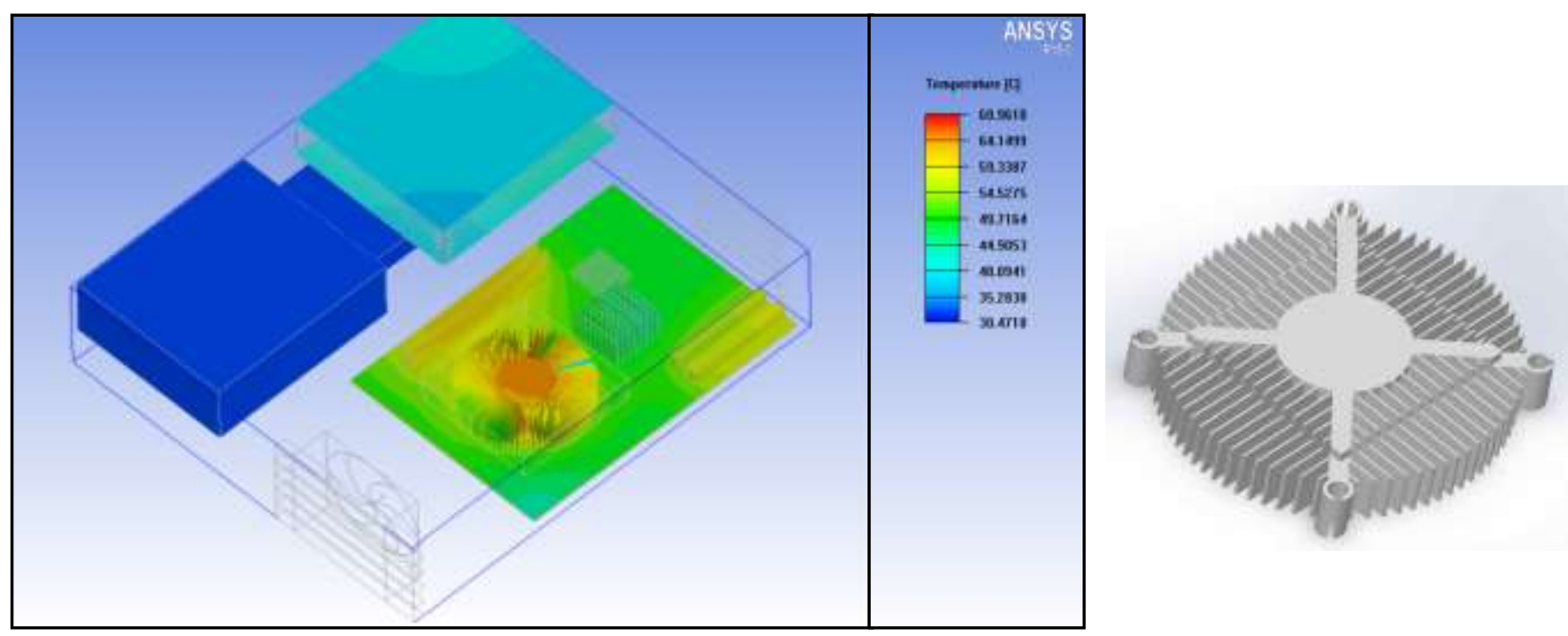

Figure 6d. Case (4).
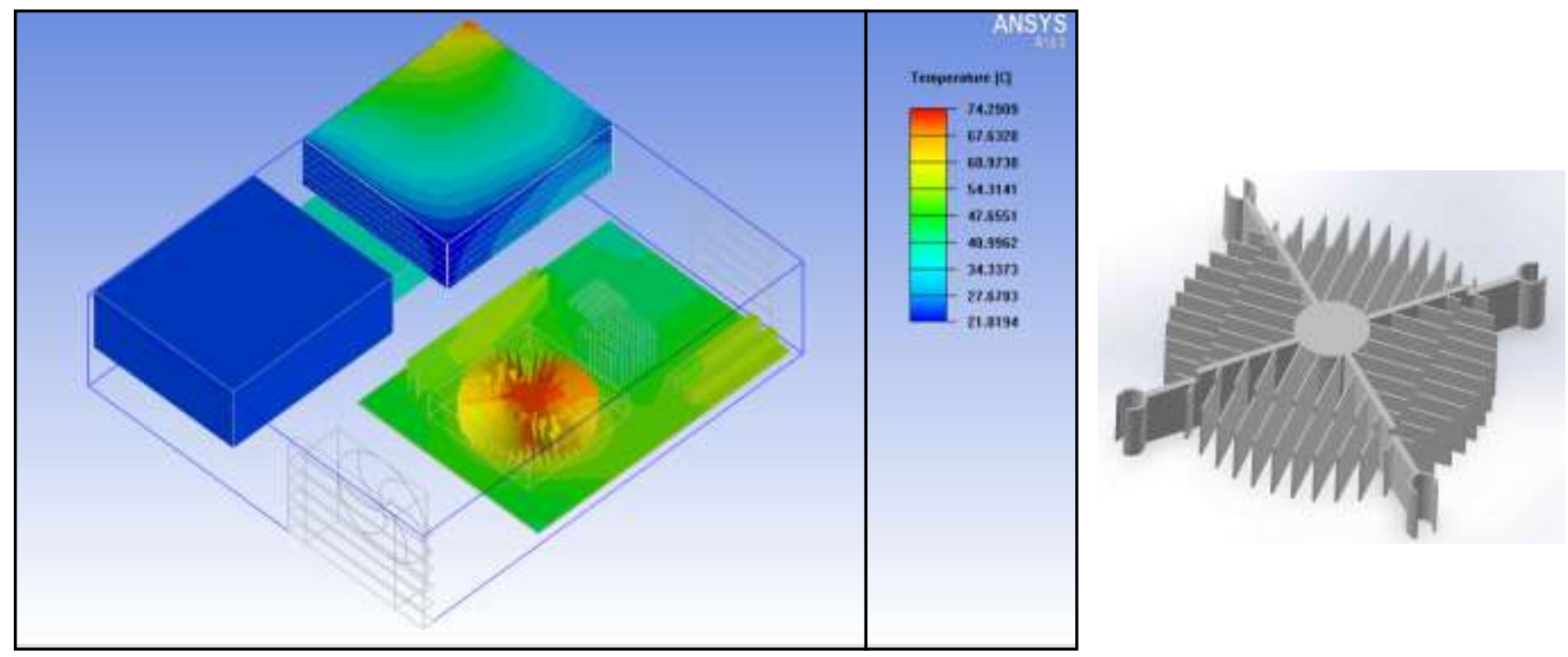
Figure 6e. Case (5).

Figure 6(a.b.c,d.e) The Temperature Contours (3D) of different models of commercial heat sink.
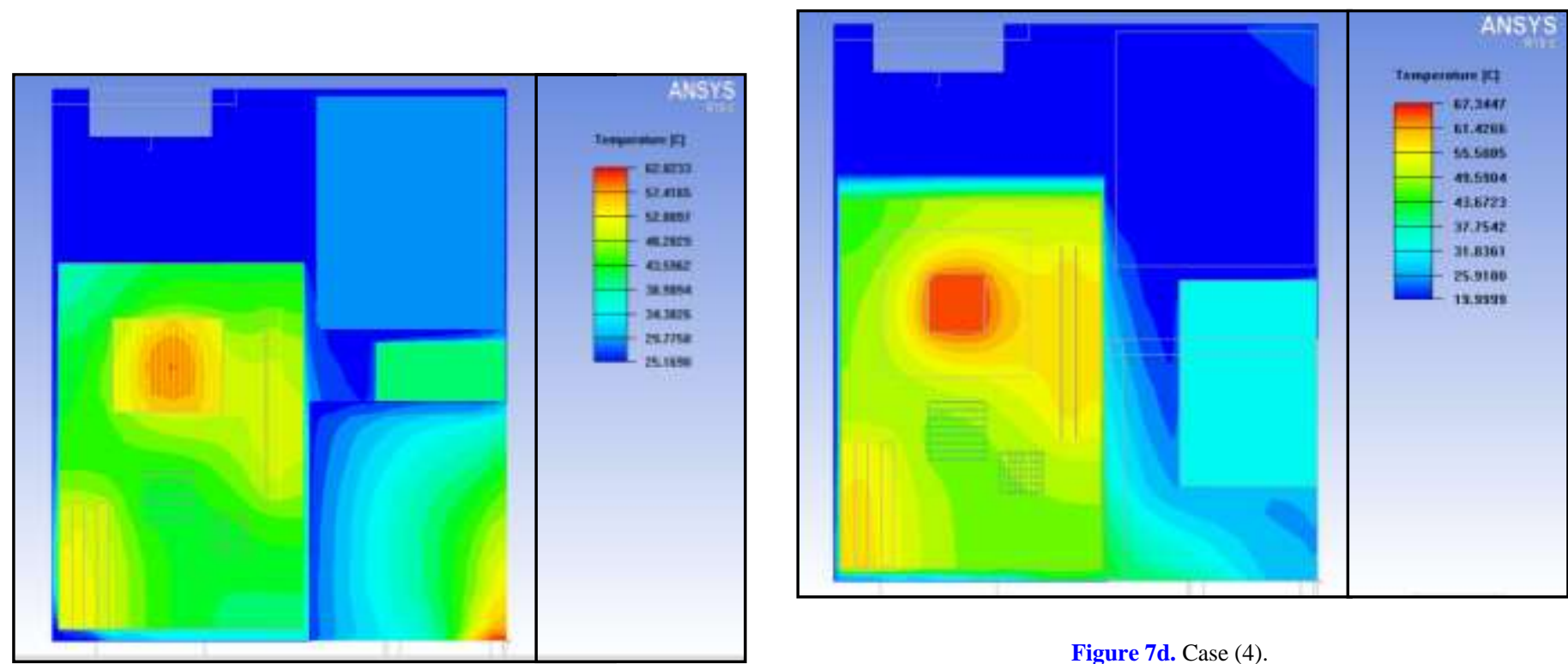

Figure 7a. Case (1).
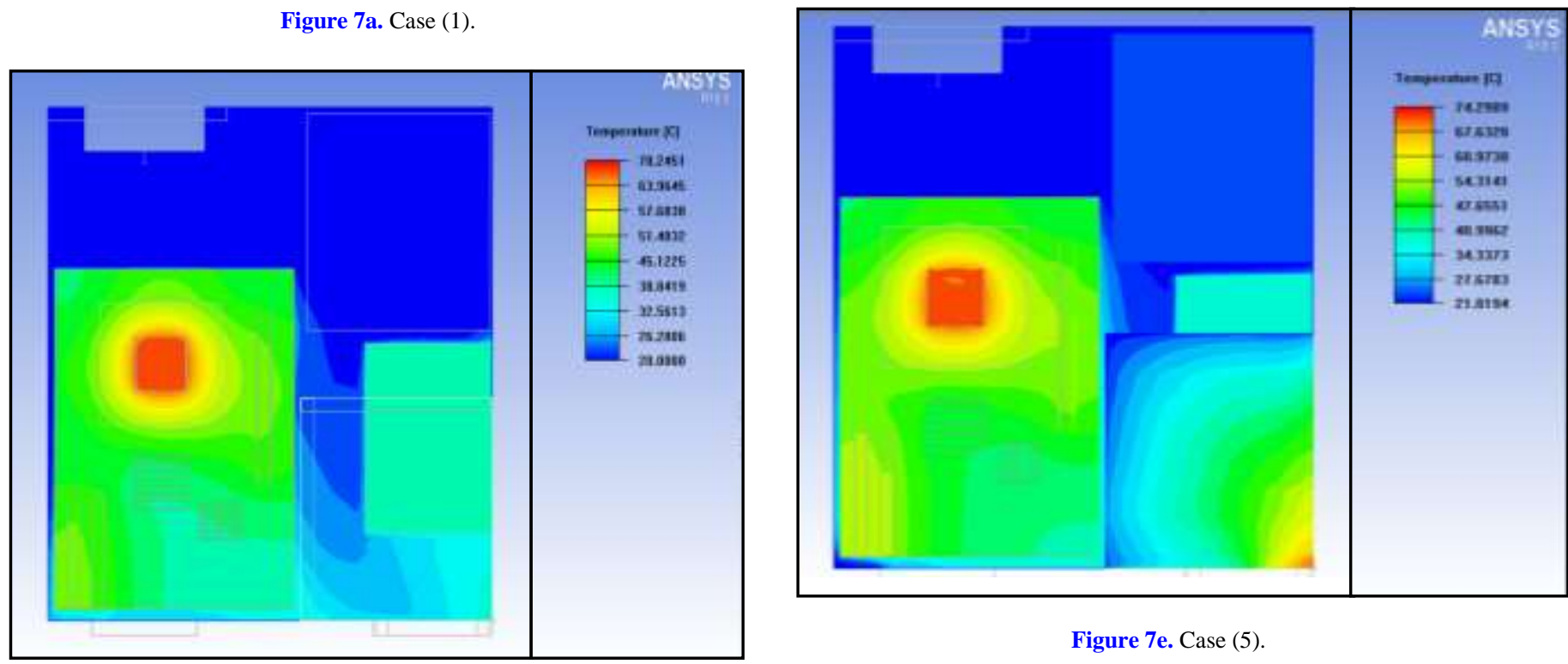

Figure 7e. Case (5).

Figure 7b. Case (2).

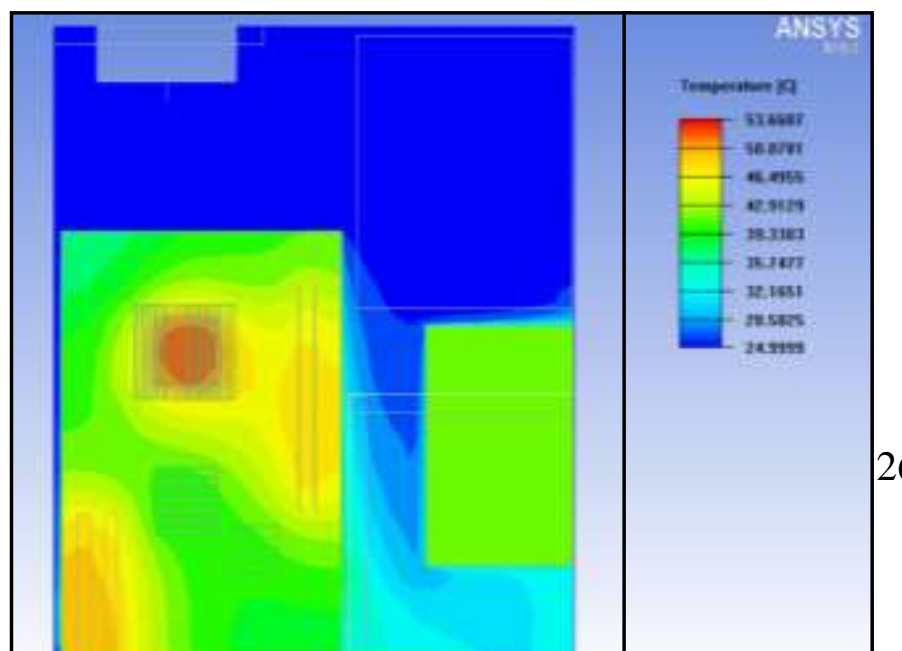

Figure 7 (a.b.c,d,e) The Temperature Contours (plan Z×X) of different models of commercial heat sink.

For all heatsinks, Its observed that the center of the heat sink is the hot spot . and there is a great decreasing of cpu temerature by about $60^{\circ} \mathrm{C}$.the most effective heat sink on cooling is the heat sink number 3 . It features the most big size, and most length fins ,its material is aluminum and has a copper heat pipes.fig. view that temperature is decresing from bottom to up. 
Figure 7c. Case (3)

the following figures view some of the characteristics for the optimum case .

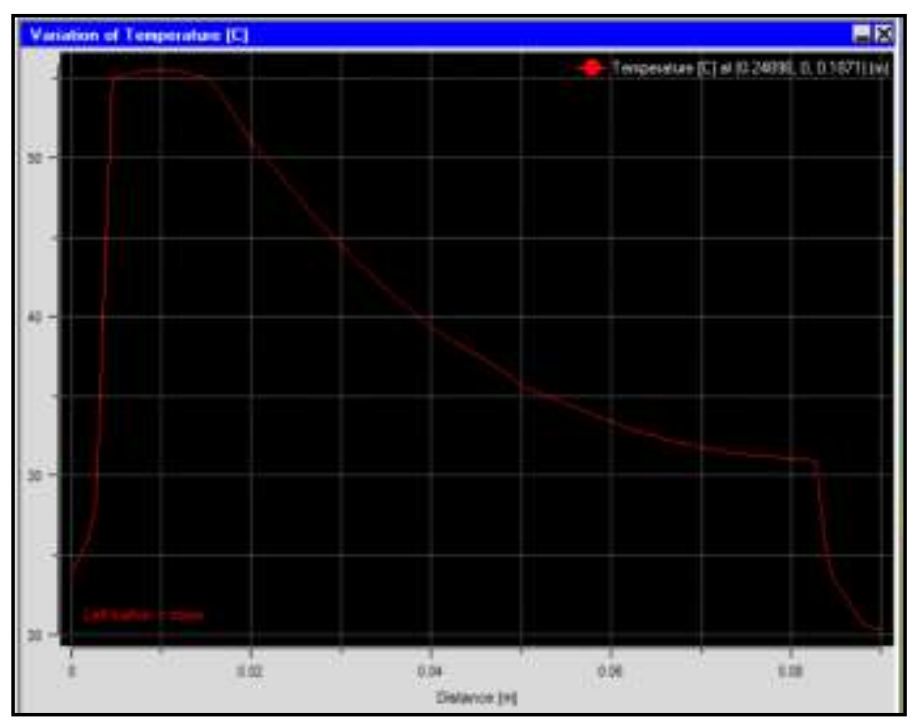

Figure 8. Relation between distance on a line passes through the center of heat sink and CPU from bottom to up and temperature

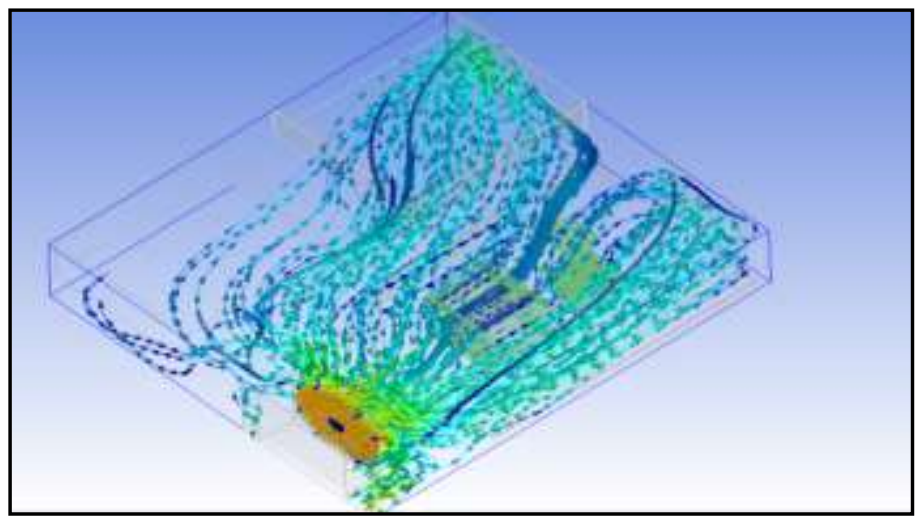

Figure 8a.

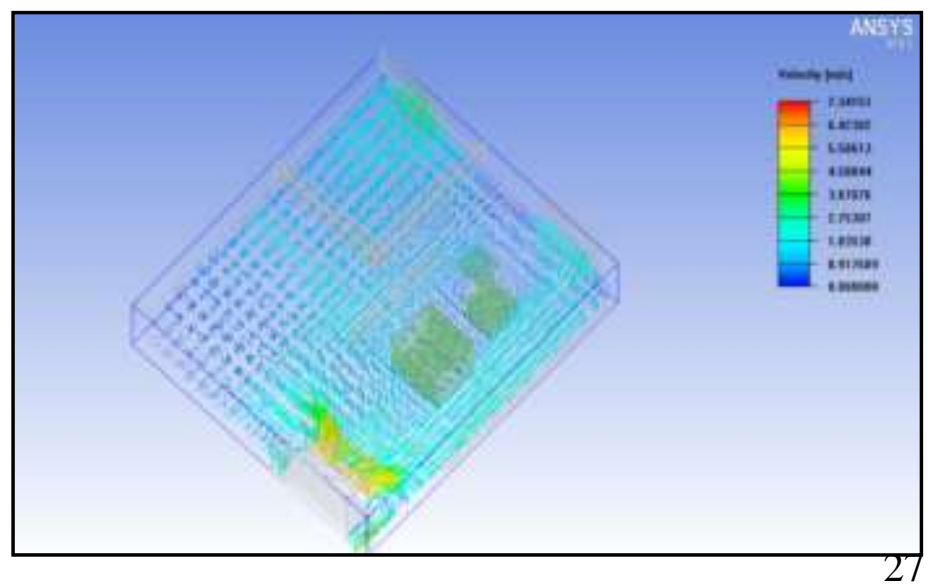

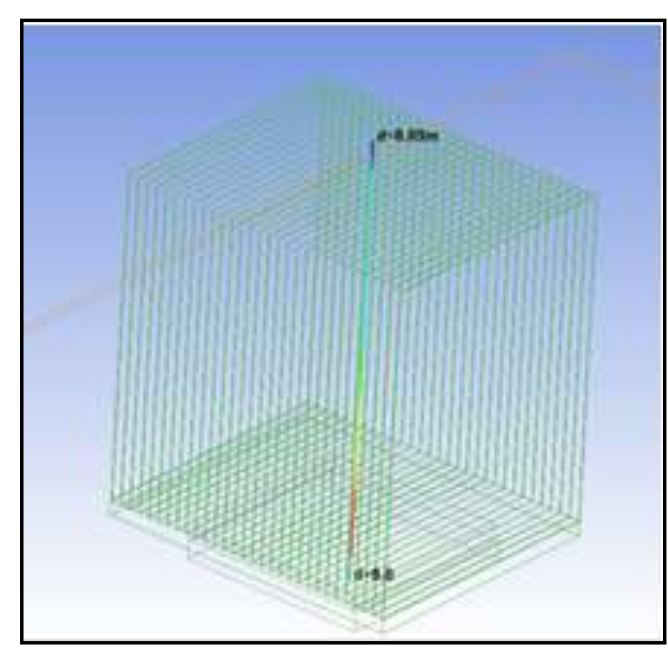

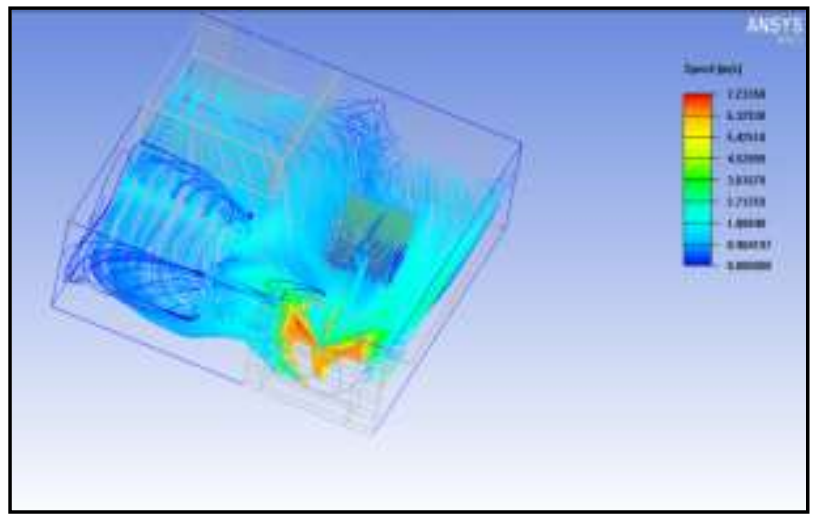

Figure 8c. 
Figure 8b.

Figure 8.(a,b,c,d) represents the fan flow, top plane of temperature distribution, speed vectors through y plane, general flow in the cabinet.

\section{CONCLUSION}

This motivation forms the basis of this study and the thesis goals are developed on this motivation. Thesis goals can be summarized as follows:

- To obtain accurate five models of different commercial heat sinks inside the cabinet and obtains CFD solutions for these models.

- To investigate the flow characteristics and cooling inside the chassis.

- $\quad$ To compare the optimum case with heat sink and without heat sink.

- To select the optimum case.

Table 4

Max and Min temp of Components

\begin{tabular}{|c|c|c|}
\hline \multicolumn{2}{|c|}{ Max and Min temp of Components } \\
\hline COMPONENT & $\begin{array}{c}\text { MAX } \\
\text { TEMP } \\
\left({ }^{\circ} \mathrm{C}\right)\end{array}$ & $\begin{array}{c}\text { MEAN } \\
\text { TEMP } \\
\left({ }^{\circ} \mathrm{C}\right)\end{array}$ \\
\hline DVD & 24.3676 & 24.3115 \\
\hline HDD & 35.5583 & 35.3983 \\
\hline CPU & 55.7918 & 54.6349 \\
\hline CPU HEAT & 55.454 & 37.4985 \\
\hline SINK & 42.6 & 40.18 \\
\hline NB HEAT SINK & 43.19 & 42.18 \\
\hline
\end{tabular}

VI. References

1. C-W Yu, R. L. Webb, "Thermal Design of a Desktop Computer System Using CFD Analysis", 17th IEEE Semi-Therm Symposium, pp. 18-26, 2001.

2. Eveloy V, Rodgers P and Hashmi MSJ (2003). Numerical prediction of electronic component heat transfer: an industry perspective. March 2003, San Jose CA, USA, 14-26 Proceedings of 19th SEMITHERM. 11-13.

3. Öztürk E (2004). CFD Analyses of heat sinks for CPU cooling with FLUENT. M.S. thesis, Middle East Technical University, Ankara, Turkey.

4. W. A. Khan,* J. R. Culham, $\dagger$ and M. M. Yovanovich (2006)

5. Ece Ayli, Caner Turk, and Selin Aradag (2013)

\begin{tabular}{|c|c|c|}
\hline $\begin{array}{c}\text { POWER } \\
\text { SUPPLY }\end{array}$ & 53.46 & 31.57 \\
\hline RAM CHIPS & 49.5 & 48.1 \\
\hline MAIN BOARD & 55.36 & 44.49 \\
\hline
\end{tabular}

Table 5

Heat Dissipation of Components

\begin{tabular}{|l|c|}
\hline Object Name & $\begin{array}{c}\text { Heat Dissipation } \\
\text { Rates (W) }\end{array}$ \\
\hline CPU & 95 \\
\hline DVD & 10 \\
\hline Hard drive & 15 \\
\hline CPU heat sink & - \\
\hline Heat sink.1 & - \\
\hline Heat sink.2 & - \\
\hline Power supply & 240 \\
\hline Mainboard & - \\
\hline RAM cards & $10 * 2$ \\
\hline PCIs & $8 * 2$ \\
\hline
\end{tabular}

6. S. Saste,H. Doshi, P. Khalate, S. Jedhe and N. Yadav "Modification in Heat Sink Design to Improve its HeatDissipation Capacity for Current CPU Design'JoEECC pp. 1-7,2018.

Nomenclature

\begin{tabular}{|c|l|}
\hline CFD & Computational fluid Dynamics \\
\hline CPU & Central processing unit \\
\hline (HxWxD) & Height, width, and Deep $(\mathrm{mm})$ \\
\hline CFM & Cubic Feet per Minute \\
\hline
\end{tabular}




\begin{tabular}{|c|l|}
\hline QRST & $\begin{array}{l}\text { Rules standing for, Quality, Resolution, } \\
\text { Smoothness and Total cell count }\end{array}$ \\
\hline $\boldsymbol{u}$ & Velocity component in x-direction, $\mathrm{m} / \mathrm{s}$ \\
\hline$\overline{\boldsymbol{V}}$ & Velocity Vector, - \\
\hline $\mathbf{v}$ & $\begin{array}{l}\text { Velocity component in y-direction, } \\
(\mathrm{m} / \mathrm{s})\end{array}$ \\
\hline $\boldsymbol{w}$ & $\begin{array}{l}\text { Velocity component in } \\
\text { (m/s) }\end{array}$ \\
\hline $\boldsymbol{f}$ & Bodirection, \\
\hline $\mathrm{P}$ & Pressure, Pa \\
\hline $\mathrm{T}$ & Temperature \\
\hline $\mathrm{T}$ & Ambient Temperature, $\mathrm{K}$ \\
\hline $\mathrm{K}$ & Thermal Conductivity, W/m.K \\
\hline$\dot{q}$ & Heat Generation rate (heat flux), W/m ${ }^{3}$ \\
\hline$\rho$ & Density, kg/m ${ }^{3}$ \\
\hline$\tau$ & Shear Stress, N/m ${ }^{2}$ \\
\hline$x, y, z$ & Position Coordinates \\
\hline
\end{tabular}

